\title{
Subliminal stimuli in the near absence of attention influence top-down cognitive control
}

\author{
Dobromir A. Rahnev • Elliott Huang • Hakwan Lau
}

Published online: 16 December 2011

(C) The Author(s) 2011. This article is published with open access at Springerlink.com

\begin{abstract}
Recent research has shown that visual stimuli can influence cognitive control functions, even if subjects are unaware of the identity of the stimuli. However, in those previous studies, subjects actively attended to the location of the subliminal stimuli. Here we assessed the role of endogenous spatial attention in such paradigms. We required subjects to quickly prepare for one of two numerical judgment tasks on the basis of the direction of motion in patches of moving dots presented in cued spatial locations. We found that irrelevant motion patches presented in the uncued spatial locations also influenced task performance. Motion in the uncued patches was weak and did not affect the perception of the cued patches. Further analyses suggested that the effect of priming by the uncued stimuli was present even for subjects who could only discriminate such stimuli at chance level. Three additional experiments confirmed that subjects paid minimal attention to the uncued locations, in that the subjects could not perform simple discriminations of conjunctions of features in those locations.
\end{abstract}

Keywords Attention - Cognitive and attentional control . Priming

Higher cognitive processes are traditionally thought to require both focused attention and awareness (Jack \& Shallice, 2001; Norman \& Shallice, 1986). Many recent empirical and theoretical studies have suggested that complex behaviors, such as cognitive control, depend on conscious attention, such that these behaviors are unlikely to be influenced by

D. A. Rahnev $(\bowtie) \cdot$ E. Huang $\cdot$ H. Lau

Department of Psychology, Columbia University,

406 Schermerhorn Hall, 1190 Amsterdam Avenue, MC 5501,

New York, NY 10027, USA

e-mail: dar2131@columbia.edu unconscious or unattended stimuli (Baars, 2002; Dehaene \& Naccache, 2001; Eimer \& Schlaghecken, 2003; Jack \& Shallice, 2001; Umiltà, 1988).

However, despite the general notion that attention and awareness are necessary for higher cognitive processing, recent studies are beginning to demonstrate that in some cases, complex behaviors can be influenced without conscious attention (Dehaene et al., 1998; Dijksterhuis, Bos, Nordgren, \& van Baaren, 2006; Ferguson \& Bargh, 2004; Mattler, 2003; Varraine, Bonnard, \& Pailhous, 2002; for reviews, see Dehaene \& Naccache, 2001; Koch, 2004; Kouider \& Dehaene, 2007). More specifically, several studies have found that cognitive control functions associated with the prefrontal cortex can be influenced by stimuli that can only be identified at chance level (we will call these stimuli "subliminal" henceforth, even though they were often not strictly subliminal in the sense that subjects could not detect their presence; instead, their identity was subliminal).

Lau and Passingham (2007) and Mattler (2003) showed that subliminal stimuli can influence task selection and preparation, one of the highest forms of cognitive control function (Koechlin, Ody, \& Kouneiher, 2003; Miller \& Cohen, 2001) known to depend on activity in the prefrontal cortex (Sakai \& Passingham, 2003). In Lau and Passingham's study, the researchers asked subjects to perform a semantic or a phonological task, depending on whether an instruction figure was a diamond or a square. The instruction figure acted as a metacontrast mask for a previously presented unconscious prime that was itself either a diamond or a square. Subjects' performance decreased when they were primed to perform the "wrong" task - that is, the task that they were not explicitly instructed to perform. This priming effect was found to be associated with task-specific activity in the prefrontal cortex; in particular, subjects 
employed the "wrong" neural resources when primed to prepare for the "wrong" task.

Furthermore, van Gaal, Ridderinkhof, Fahrenfort, Scholte, and Lamme (2008) showed that inhibitory control, another form of higher cognitive control, could be influenced by stimuli presented below the threshold of conscious perception. The researchers demonstrated that metacontrastmasked unconscious no-go signals increased reaction times (RTs) and led to higher percentages of response terminations. In addition, electroencephalographic recordings showed that the no-go signal produced activations in the prefrontal cortex. These activations were found to be responsible for the inhibitory control and independent of the visual processing of the stimulus.

Two more studies from the same group further demonstrated the power of subliminal stimuli to influence cognitive control functions. Van Gaal, Ridderinkhof, van den Wildenberg, and Lamme (2009) found that subliminal stop signals decreased response speed. This effect was particularly strong for the subjects who were best able to inhibit their action when given a consciously perceived stop signal. Finally, van Gaal, Ridderinkhof, Scholte, and Lamme (2010) demonstated that subliminal no-go signals exert inhibitory control by activating prefrontal regions such as the inferior frontal cortex and presupplementary motor area that themselves correlate with the slowing down of RTs.

Taken together, these studies provide strong evidence that subliminal stimuli can influence cognitive control. However, in the studies mentioned above (Lau \& Passingham, 2007; Mattler, 2003; van Gaal et al., 2008; van Gaal et al., 2010; van Gaal et al., 2009) the invisible primes were presented in the same spatial location as the target, and thus subjects actively attended to the location of the primes. Thus, it remains unclear whether stimuli that do not receive spatial attention can produce the same effect.

It is widely assumed that certain kinds of priming are impossible without attention (Dehaene, Changeux, Naccache, Sackur, \& Sergent, 2006; Koch \& Tsuchiya, 2007). For example, it is widely thought that visual stimuli are processed in parallel in early visual areas, but that only attended stimuli are able to go through an attentional bottleneck and influence higher cognitive areas such as the prefrontal cortex (Lachter, Forster, \& Ruthruff, 2004; Wolfe \& Horowitz, 2004). Some researchers have specifically argued that unattended stimuli can be processed in early visual areas but cannot reach areas that code for the semantic properties of objects (Lachter et al., 2004). Evidence for such conclusions comes from various studies in which subjects have failed to identify words or letters without focused attention (Besner, Risko, \& Sklair, 2005; Lachter et al., 2004; Marzouki, Grainger, \& Theeuwes, 2007).

Here we demonstrate that priming of cognitive control is possible without focused top-down attention. We presented motion primes that were outside the focus of attention. To anticipate the results, we found that the primes influenced task preparation and execution, suggesting that endogenous spatial attention may not be necessary for the priming of cognitive control.

\section{Experiment 1}

We investigated whether subliminal stimuli outside of the focus of attention can influence higher-order cognitive functions. To this end, we placed the primes in the periphery, rather than at fixation, and cued subjects to attend to locations away from the primes.

Most previous studies on subliminal influences on higher cognitive functions have used color or simple geometric forms (Lau \& Passingham, 2007; van Gaal et al., 2008; van Gaal et al., 2010; van Gaal et al., 2009). However, such stimuli might not be optimized for processing in the periphery (McMullen, MacSween, \& Collin, 2009; Moreland, Jameson, \& Hurvich, 1972). Therefore, we decided to use motion stimuli, since the presence of motion improves vision for stimuli presented peripherally (Finlay, 1982) and thus increases the chances of finding priming from peripheral stimuli.

\section{Method}

Subjects A group of 21 students from the Columbia University undergraduate population (11 women, 10 men; mean age $=22.4$ years, range $=18-30$ ) participated in this experiment and were paid $\$ 10$ for approximately $1 \mathrm{~h}$ of participation. Two of the subjects were excluded because they responded to the tasks in the main part of the experiment almost completely randomly. All subjects had normal or corrected-to-normal vision and had signed an informed-consent statement. The research was approved by Columbia University's Committee for the Protection of Human Subjects.

Materials and procedure Four black circles $\left(0.04 \mathrm{~cd} / \mathrm{m}^{2}\right.$, visual angle $=2.5 \mathrm{deg}$ ) were presented in the four quadrants of the screen with the center of each circle located $4.6 \mathrm{deg}$ away from fixation (Fig. 1). This configuration was chosen in order to minimize eye movements, as has been done in previous studies (Bahrami, Lavie, \& Rees, 2007). White (27 $\mathrm{cd} / \mathrm{m}^{2}$ ) arrows pointed along one of the two diagonals. The stimuli were presented on a dark blue background $(2.5 \mathrm{~cd} /$ $\mathrm{m}^{2}$ ). Subjects were seated in a dimmed room about $50 \mathrm{~cm}$ away from the computer monitor and instructed to maintain fixation on a central red $\operatorname{dot}\left(6.6 \mathrm{~cd} / \mathrm{m}^{2}\right)$ displayed on top of the white arrow for the duration of each trial. Stimuli were generated using Psychophysics Toolbox (Brainard, 1997) in MATLAB (The MathWorks, Natick, MA) and were shown 


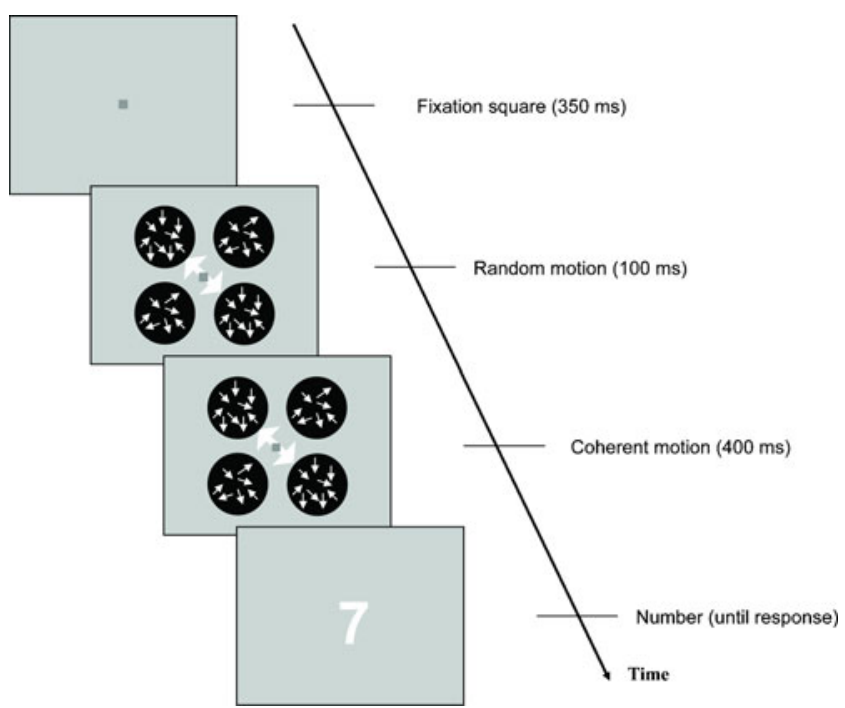

Fig. 1 Experimental procedure. Each trial began with $350 \mathrm{~ms}$ of fixation. This was followed by $100 \mathrm{~ms}$ in which the dots in the cued diagonal moved randomly, while the dots in the uncued diagonal had a coherence of $4 \%$. Then, for $400 \mathrm{~ms}$ the dots in the cued diagonal moved with a subject-specific coherence that was clearly visible to subjects (mean coherence $=33 \%$ ), while the dots in the uncued diagonal kept the same coherence of 4\%. Each trial ended with the presentation of a single-digit number until a response was provided. Subjects needed to indicate whether the number was greater or less than 5 if the cued motion was upward, and to indicate whether the number was odd or even if the cued motion was downward

on an iMac monitor (LCD, 24-in. monitor size, 1,920 $\times$ 1,200 pixel resolution, $60-\mathrm{Hz}$ refresh rate) that produced no blurring of the motion stimuli.

The trials in the main task of the experiment began with $350 \mathrm{~ms}$ of fixation, followed by a 500-ms presentation of the four circles. White arrows randomly cued one of the diagonals on each trial. The arrows were presented simultaneously with the circles and remained on screen for the full 500 $\mathrm{ms}$ of the presentation. Within each circle were moving white dots (density $=2.4 \mathrm{dots} / \mathrm{deg}^{2}$, speed $=9 \mathrm{deg} / \mathrm{s}$ ) with varying levels of coherence. During the first $100 \mathrm{~ms}$ of the presentation, the dots in the cued diagonal moved randomly (coherence $=0 \%$ ) and subsequently moved with a coherence predetermined (see below) for each subject for $400 \mathrm{~ms}$. The dots in the uncued diagonal always had a coherence of $4 \%$, which was found to produce performance around chance level in a pilot study. Subjects were instructed to pay attention to the direction of the overall motion of the dots in the cued diagonal and to disregard the motion in the uncued diagonal.

The presentation of the moving dots was immediately followed by a single-digit number (height $=6.6 \mathrm{deg}$ of visual angle) presented at fixation. Depending on the direction of motion of the dots in the cued diagonal, subjects needed to do one of two tasks. If the direction of motion was upward, subjects needed to compare the magnitude of the subsequent number with 5 (larger or smaller); if the direction was downward, they needed to indicate whether the number was odd or even. The "A" key was used to indicate that numbers were less than 5 or odd, while the "L" key was used for numbers that were greater than 5 or even. The digit 5 was never presented. Feedback was given for wrong answers in the form of a large red " $\mathrm{X}$ " (height $=6.6 \mathrm{deg}$ of visual angle). The numbers $1,3,6$, and 8 required the same motor response, regardless of the direction of the dot motion; therefore, we refer to these numbers as "no-conflict" numbers. On the other hand, the numbers 2, 4, 7, and 9 required different motor responses depending on the direction of the dot motion, and are thus referred to as "conflict" numbers. On two-thirds of the trials, the subsequent number was chosen randomly from the conflict numbers, and on one-third of the trials, it was chosen randomly from the noconflict numbers. Subjects were encouraged to answer as quickly as possible but without compromising accuracy.

The experiment began with a calibration procedure that ensured that subjects clearly saw the direction of the motion in the cued diagonal. Subjects first experienced a coherence level of $25 \%$ in the cued diagonal and were required to complete 50 trials, indicating only the direction of the motion in that diagonal. If their performance was lower than $90 \%$ correct, the coherence level was increased in increments of $5 \%$ until the subject reached a performance of $90 \%$ correct or better (in 50 trials). This obtained value for the coherence level was used in the rest of the experiment. For two of the subjects, $25 \%$ coherence was used, and a visibility test was performed at the end of the experiment to confirm that they could clearly see the motion in the cued diagonal. The resulting mean coherence for all subjects was $33.3 \%$; the range was $25 \%$ to $65 \%$.

The main part of the experiment consisted of 800 trials separated into four runs. Each run consisted of four blocks of 50 trials. The directions of motion in both diagonals were randomized on each trial and thus were independent of each other. This resulted in the motion in the cued diagonal being the same as the motion in the uncued diagonal on approximately half of the trials. We call these trials "congruent," while the trials on which the two directions were opposite we call "incongruent." We predicted shorter RTs and higher accuracy for congruent than for incongruent trials, and thus used one-tailed paired $t$ tests to test these predictions.

To test the extent to which the subjects were conscious of the direction of motion in the uncued diagonal, we performed one simple task at the end of the experiment, which we refer to as the "visibility test." Subjects were presented with the same trial presentation as in the calibration procedure and in the main part of the experiment, but this time they needed to report first the direction of motion in the cued diagonal and then the direction of motion in the uncued diagonal. Subjects completed 100 trials of this test. It should 
be noted that our test of visibility was conservative. In the main part of the experiment, the motion in the uncued diagonal was completely irrelevant to the task and subjects were instructed to ignore it, while in the visibility test, subjects were encouraged to pay attention to both diagonals. Therefore, it was likely that subjects' performance at indicating the direction of motion in the uncued diagonal in the visibility test would overestimate their level of awareness of the motion in that diagonal during the main part of the experiment.

\section{Results and discussion}

In order to minimize the effects of trials with extreme RTs, we excluded trials with RTs below $250 \mathrm{~ms}$ or above $8 \mathrm{~s}$ (resulting in $1 \%$ of trials being excluded) and used median rather than mean RTs in our analyses.

We tested whether the RTs and the proportions of correct answers differed between the congruent and incongruent trials (i.e., trials on which the cued and uncued motions were in the same or in different directions, respectively). A paired-samples $t$ test showed that subjects' accuracy was higher on congruent than on incongruent trials $[t(18)=$ 2.67, $p=.008$, one-tailed $t$ test; Fig. 2]. Another pairedsamples $t$ test showed that the subjects' RTs on the congruent trials were significantly shorter than those on the incongruent trials $[t(18)=2.06, p=.027$, one-tailed $t$ test $]$. The test on RTs became marginally significant if we used a twotailed $t$ test $(p=.054)$, but we felt that a one-tailed test was more appropriate because of our clear prediction. Taken together, the results on accuracy and RT showed that subjects' performance was influenced by the motion that was outside the focus of their spatial attention.

However, one could argue that these effects were due to the subjects getting perceptually distracted by the incongruent motion in the uncued diagonal. To rule out this possibility, we analyzed the data from the calibration procedure (i.e., the initial stage of the experiment in which we determined the level of motion coherence for each subject). We found that the uncued motion, regardless of whether it was congruent to the cued motion, did not affect the accuracy in discriminating the cued motion $(p>.1)$. Similarly, we analyzed the data from the visibility test (i.e., the 100-trial procedure performed after the main task in which we explicitly tested for the visibility of the uncued motion patches). Again, we found no significant difference in accuracy for indicating the direction of motion on the cued diagonal, regardless of whether the uncued motion was congruent or incongruent with the cued motion.

Further analysis of the results from the main task of the experiment also suggested that the priming from the uncued stimuli was unlikely to operate at a perceptual level. Specifically, we tested whether the incongruency effect reported above was bigger for the trials on which both task sets required the same keypress (no-conflict trials) or the trials on which the two task sets required different keypresses (conflict trials). If our subjects were simply distracted perceptually by the incongruent uncued motion, one might expect the decreases in accuracy from congruent to incongruent trials to be the same for conflict and no-conflict trials. A repeated measures analysis of variance with the factors Stimulus Congruency and Response Conflict showed an interaction between response conflict and stimulus congruency, such that the difference in subjects' accuracies on congruent and incongruent trials was significantly bigger for conflict than for no-conflict numbers $[F(1,18)=6.3, p$ $=.02]$. A similar test for RTs did not show significant results. However, since the no-conflict trials did not necessarily require the processing of the cued motion (one could do the number task even without knowing which task he or she was supposed to do), the above analysis cannot conclusively rule out the possibility that the uncued stimuli led to perceptual distraction. Nevertheless, taken together, the analyses of the calibration procedure, the visibility test,

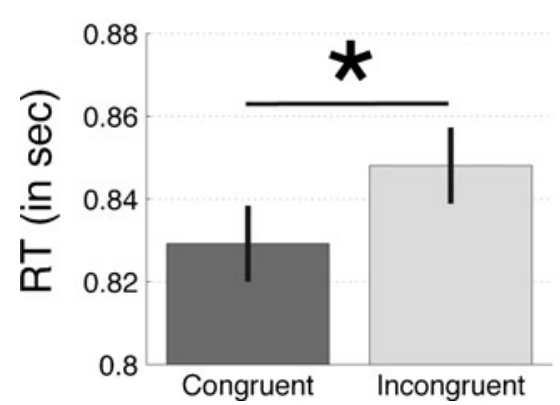

Fig. 2 Experiment 1: Reaction times (RTs) and $d^{\prime}$ for trials in which the uncued motion was congruent or incongruent with the motion in the cued diagonal. Left panel: RTs for congruent trials were significantly shorter than those for incongruent trials $(p=.027)$. Right panel:

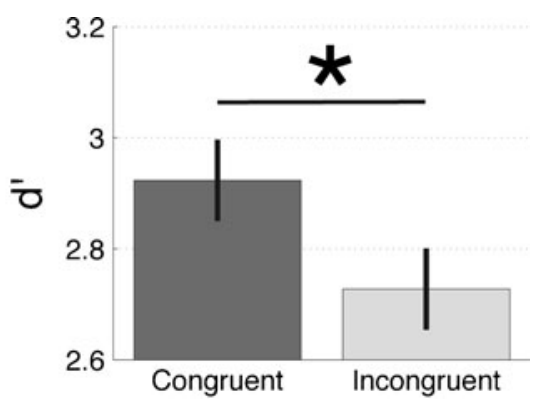

$d^{\prime}$ was significantly higher for congruent than for incongruent trials ( $p$ $<.01)$. These data suggest that the uncued motion primed subjects' task sets. Error bars are within-subjects standard errors of the means 
and the conflict/no-conflict trials suggested that the priming effect was likely to act via higher cognitive processes such as task preparation or response processing, rather than via low-level perceptual processes.

Next, we tested whether our subjects were aware of the direction of motion in the uncued diagonal. We found that subjects were poor at indicating the direction of motion in the uncued diagonal during the visibility test $\left(d^{\prime}=0.27\right)$ but were nevertheless significantly better than chance $(p<.05$; chance level: $d^{\prime}=0$ ). Therefore, in order to test whether the uncued motion could influence subjects even in the absence of awareness, we performed a median split of the subjects in terms of their $d$ 's in the visibility test and analyzed the half with lower $d$ 's. These 9 subjects were at chance when discriminating the direction of motion in the uncued diagonal during the visibility test (mean $d^{\prime}=-0.04, p=.81$ ). Despite that, these subjects were still significantly faster on congruent than on incongruent trials $[t(8)=1.99, p=.04]$, providing some evidence that unconscious stimuli outside of the focus of spatial visual attention can still influence task set. We obtained similar results when we excluded all subjects with positive $d$ 's or all subjects with performance better than $55 \%$ on the visibility test (both $p$ s $<.05$ ).

To provide a further test as to whether the effect we observed was likely to hold for uncued stimuli that could only be identified at chance level, we performed an analysis developed by Greenwald, Draine, and Abrams (1996). We analyzed the magnitude of the RT effect in the main part of the experiment as a function of performance on the prime perceptibility task in the visibility test (Fig. 3). We performed a linear fit and excluded 2 subjects who emerged as significant outliers (Barnett \& Lewis, 1994). The zerointercept on the $y$-axis of the linear fit was significantly

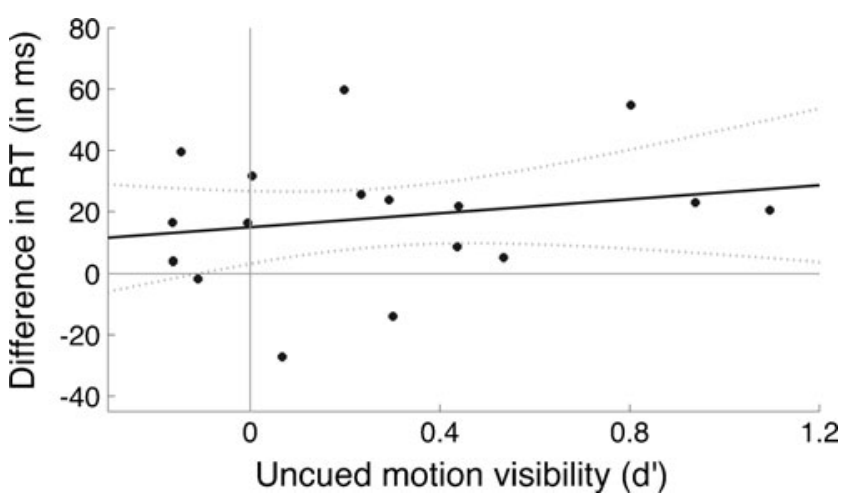

Fig. 3 Experiment 1: Magnitude of the reaction time (RT) effect as a function of performance on the direct measure of prime perceptibility. Each point on the scatterplot represents an individual subject. The central line is the best linear fit with $90 \%$ confidence intervals. The zero-intercept was significantly positive $(p<.05)$, indicating that even when the primes could only be identified at chance level, there was still a significant effect on task preparation positive (intercept $=14.9 \mathrm{~ms}, p<.05$ ), suggesting that even when subjects were at chance in determining the direction of motion in the uncued diagonal, that motion still had a significant effect on task set. Not excluding the 2 outliers led to an even higher intercept value $(20.6 \mathrm{~ms})$; however, their inclusion increased the variance of the estimate of the intercept by $70 \%$.

\section{Experiment 2}

Experiment 1 suggested that weak stimuli that are outside of the focus of attention can still influence such higher cognitive functions as task setting. However, one could argue that even though subjects were instructed not to attend to the uncued diagonal, they nevertheless did so. This was unlikely for several reasons. First, we explicitly instructed subjects not to attend to the uncued motion patches. Second, these motion patches were irrelevant to the subjects' task, so devoting attentional resources to their processing could only hurt performance. Third, the uncued stimuli were weak and therefore could attract only limited exogenous attention.

Nevertheless, since establishing that subjects did not pay focused attention to the uncued stimuli was central to Experiment 1 , we decided to further investigate this issue. To this end, in Experiment 2 we required subjects to perform the task from Experiment 1 but changed the stimuli in the uncued locations. Rather than weak motion, we used redgreed and green-red disks (see Fig. 4a and b). It has previously been demonstrated that processing of such stimuli requires spatial attention (Lee, Koch, \& Braun, 1999; Li, VanRullen, Koch, \& Perona, 2002). In particular, Li et al. presented these stimuli in the periphery while subjects were performing a task at fixation. The researchers found that subjects were at chance at discriminating between the two kinds of disks, while they were able to discriminate well between pictures that contained faces or animals.

Given that such colored disks have been shown to require focused attention to be processed ( $\mathrm{Li}$ et al., 2002), these stimuli provide an opportunity for us to test the level of attention deployed to the uncued diagonal in Experiment 1. If subjects indeed paid focused attention to the uncued locations, they should be able to discriminate between red-green and greed-red disks when these are placed in the uncued diagonal. On the other hand, if subjects were not able to pay substantial attention to the uncued stimuli, they should be at chance at discriminating the colored disks. In Experiment 2, we adjudicated between these two possibilities. To anticipate, we found that subjects were at chance at identifying the colored disks when simultaneously performing the task from Experiment 1, but they were able to identify the disks when they paid focused attention to them (without doing a concurrent task). 


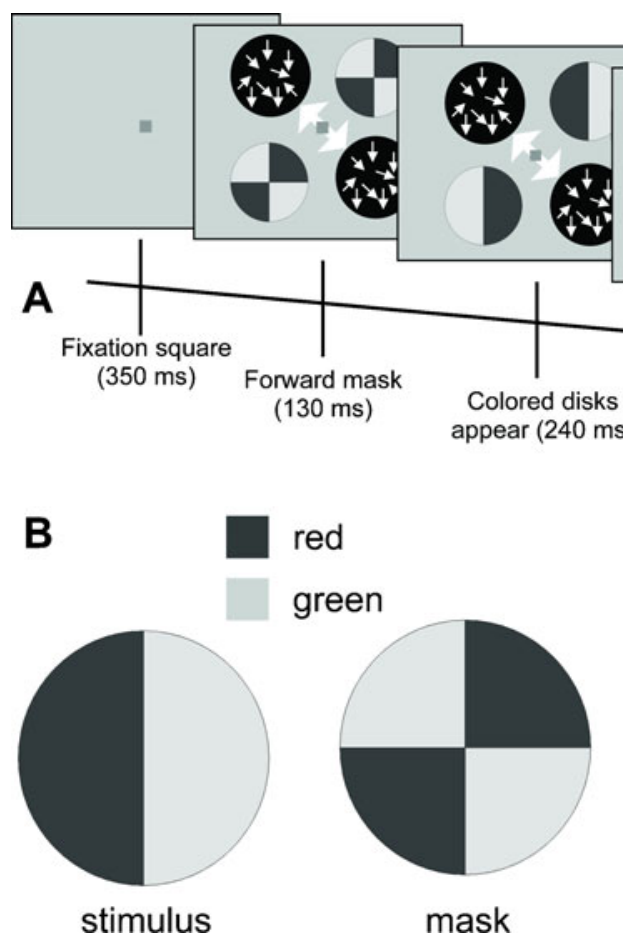

Fig. 4 Experiment 2: Task and results. (a) The task used in Experiment 2 was very similar to that used in Experiment 1, with the difference that in the uncued locations we presented colored stimuli with no motion. The colored stimuli were red-green circles, masked by colored pie circles with alternating colors. The durations of the stimuli were chosen individually for the same subject, but the forward and backward masks had equal durations that always produced a total duration of $500 \mathrm{~ms}$ for the stimulus presentation. (b) The stimuli placed in the uncued diagonal are shown. Subjects' task was to disregard the masks to indicate whether or not the two circles placed in the uncued

\section{Method}

Subjects A group of 10 students from the Columbia University undergraduate population (6 women, 4 men; mean age $=20.5$ years, range $=18-27)$ participated in this experiment and were paid $\$ 10$ for approximately $1 \mathrm{~h}$ of participation. One of the subjects had participated in Experiment 1 as well. All subjects had normal or corrected-to-normal vision and had signed an informed-consent statement.

Materials and procedure The main task was the same as in Experiment 1. However, rather than moving dots, the uncued circles now contained red-green or green-red disks (Fig. 4b). Each of the uncued circles from Experiment 1 was replaced by a single colored disk, so the overall the presentation included two colored disks, one in each of two diagonally opposite quadrants of the screen. We designed this experiment according to the paradigm developed by $\mathrm{Li}$, VanRullen, Koch, and Perona (2002), in which the researchers established that little attention was paid to a peripheral location when a demanding central task was presented. The colored disks were vertically separated into two regions of

locations had the same configuration (i.e., whether the left sides of the circles had the same color). We used these stimuli because it had previously been shown that their identification requires attention (Lee et al., 1999; Li et al., 2002). (c) Subjects were able to perform the task on the uncued color stimuli when they paid undivided attention to them, as indicated by the "single task" bar. However, when subjects were required to simultaneously perform the number task from Experiment 1 , their performance was not better than chance ("dual task" bar), suggesting that the number task does not leave attentional resources available to "spill" to the uncued locations

different colors. On half of the trials, the left region was green, and on the other half the left region was red. These stimuli were forward and backward masked by a pie separated into four quadrants (Fig. 4a). The mask had one of two possible configurations. In one configuration, the first and third quadrants of the mask were green and the second and fourth quadrants were red. In the other configuration, the first and third quadrants were red and the second and fourth quadrants were green. The configurations of the forward and backward masks were randomly chosen on each trial, so about half of the time they were the same and half of the time they were different.

The experiment started with a brief training with what we called the "color task." Subjects were asked to attend to the two colored disks that appeared in the otherwise uncued diagonal and indicate whether the two disks had the same configuration or not (same-different task). For example, if one of the disks was green-red (left part green, right part red), the subjects needed to indicate "same" if the other disk was also green-red and "different" if the other disk was redgreed. The duration of the presentation of the colored disks was adjusted for each subject individually so that they could 
achieve at least $70 \%$ correct performance on the same-different task (mean duration $=240 \mathrm{~ms}$ ). The forward and backward masks had equal lengths so that the whole presentation was $500 \mathrm{~ms}$ (mean duration of each mask $=130 \mathrm{~ms}$ ).

The experiment proceeded with a calibration procedure that was the same as in Experiment 1. This procedure identified the proportion of coherent moving dots for each subject and ensured that subjects could correctly identify the direction of motion in the cued diagonal on at least $90 \%$ of trials.

Finally, the main task was similar to the one in Experiment 1: Subjects needed to do the number task on the basis of the direction of motion in the cued diagonal. However, in this experiment we asked subjects to complete the samedifferent task right after indicating their answer on the number task. In other words, subjects were completing a dual task. This part of the experiment consisted of 400 trials separated into four blocks of 100 trials. We analyzed the performance on the same-different task in this part of the experiment to test whether subjects had attentional resources that "spilled" to the uncued diagonal.

\section{Results and discussion}

As in Experiment 1, we minimized the effects of trials with extreme RTs by excluding those with RTs below $250 \mathrm{~ms}$ or above $8 \mathrm{~s}$ (resulting in $2 \%$ of trials excluded) and using median rather than mean RTs in our analyses.

We first tested whether there were significant differences between Experiments 1 and 2 in the main task. Subjects were able to judge the direction of motion in the cued diagonal equally well in the two experiments $[t(27)=0.04$, $p=.97]$. Furthermore, they performed equally well on the number task $[t(27)=0.24, p=.81]$. The only difference came from the RTs, which were higher in Experiment 2 (mean $\mathrm{RT}=1.36 \mathrm{~s}$ ) than in Experiment 1 (mean RT $=$ 0.84 s) $[t(27)=3.31, p=.003]$. This difference was to be expected, given that in Experiment 2 subjects were performing a dual task and in Experiment 1 they were supposed to ignore the uncued stimuli and perform a single task on the basis of the cued motion.

We then turned to testing the main effect of interest. Not surprisingly, subjects were able to indicate whether the two disks were the same or different in the color task when they paid attention to the disks and ignored the coherent motion $[t(9)=7.68, p<.0001$; see Fig. 4c]. Critically, subjects were not able to distinguish between the colored disks in the main task of the experiment, where they were concurrently performing the same number task from Experiment 1 [mean accuracy $=53.8 \%$ correct; $t(9)=1.53, p=.16]$. The fact that RTs for the number task were significantly higher in this experiment as compared to Experiment 1 shows that subjects were actively engaging with the second task and that their chance performance was not due to ignoring that task. In other words, the number task was so attentionally demanding that subjects were unable to do a task in the uncued diagonal known to require focused attention (Lee et al., 1999; Li et al., 2002), even when they were actively attempting to perform well on both tasks.

\section{Experiment 3}

The results of Experiment 2 suggested that in the paradigm from Experiment 1, subjects were unlikely to have been able to pay focused attention to the uncued stimuli. This conclusion is strengthened by the fact that unlike the uncued motion in Experiment 1, the colored disks in Experiment 2 were task relevant. This means that in Experiment 1 the best strategy for subjects was to ignore the uncued motion, while in Experiment 2 subjects' best strategy was to try to pay some attention to the colored disks. In other words, subjects probably paid more endogenous attention to the colored disks than to the uncued motion, yet this was insufficient to perform the simple task of identifying the red-green discs.

Nevertheless, it could be argued that the static disks in Experiment 2 might not have attracted exogenous attention as much as the uncued moving dots in Experiment 1 (Jonides, 1981). Therefore, to control for the amount of exogenous attention attracted by the stimuli in the uncued diagonal, in Experiment 3 we placed motion stimuli in the uncued locations. As in Experiment 2, we asked subjects to complete a same-different task based on the colors red and green; here, we used red and green moving dots and had subjects identify the direction of motion for each color. To anticipate, we found that the subjects were able to identify the motion direction of the colored dots when they paid focused attention to them; however, when subjects were simultaneously engaged in the main task from Experiment 1 , performance for the colored dots fell to chance level.

\section{Method}

Subjects A group of 9 students from the Columbia University undergraduate population ( 5 women, 4 men; mean age $=22.9$ years, range $=18-30)$ participated in these experiment and were paid $\$ 10$ for approximately $1 \mathrm{~h}$ of participation. One of the subjects had participated in Experiments 1 and 2, and another had participated in Experiment 2. All subjects had normal or corrected-to-normal vision and had signed an informed-consent statement.

Materials and procedure The main task was the same as in Experiments 1 and 2. As in Experiment 2, we asked subjects to do a second task right after completing the number task. However, instead of masked red-green disks, we presented 
red and green coherent motion in the uncued locations (Fig. 5a and b). The motion had exactly the same parameters as the motion in Experiments 1 and 2 (density $=2.4$ dots/ $\mathrm{deg}^{2}$, speed $=9 \mathrm{deg} / \mathrm{s}$ ). The red dots could occupy either the left or the right side of the disk and moved either up or down. The green dots occupied the other side and moved in the opposite direction. The side and direction of motion of the red dots were chosen randomly for each of the two circles on each trial. Subjects' task was to indicate whether the red dots were moving in the same direction in the two circles located in the uncued diagonal. The dots were presented for the full $500 \mathrm{~ms}$, and unlike in Experiment 2, we did not mask the colored stimuli. Instead, we adjusted the coherence of the moving dots for each subject individually so that the subjects were able to accurately perform the same-different task at least $70 \%$ of the time (mean coherence $=29 \%$ ).

Everything else in this experiment was similar to Experiment 2. Briefly, the experiment began with a brief training with what we called the "color task," in which the coherence of the colored dots was adjusted for each subject individually. We proceeded with the calibration procedure that ensured that subjects could correctly identify the direction of motion in the cued diagonal on at least $90 \%$ of trials. Finally, the main task was the same as in Experiment 2. The subjects needed to do the number task on the basis of the direction of motion in the cued diagonal and to complete the same-different task right after indicating their answer on the number task. This part of the experiment consisted of 400 trials separated into four blocks of 100 trials.

\section{Results and discussion}

As in Experiments 1 and 2, we minimized the effects of trials with extreme RTs by excluding trials with RTs below $250 \mathrm{~ms}$ or above $8 \mathrm{~s}$ (resulting in $2 \%$ of trials excluded) and using median rather than mean RTs in our analyses.

As in Experiment 2, we tested whether any significant differences appeared between Experiments 1 and 3 in the main task. The differences mirrored the ones from Experiment 2. Comparing Experiments 1 and 3, we found that subjects were able to judge the direction of motion in the cued

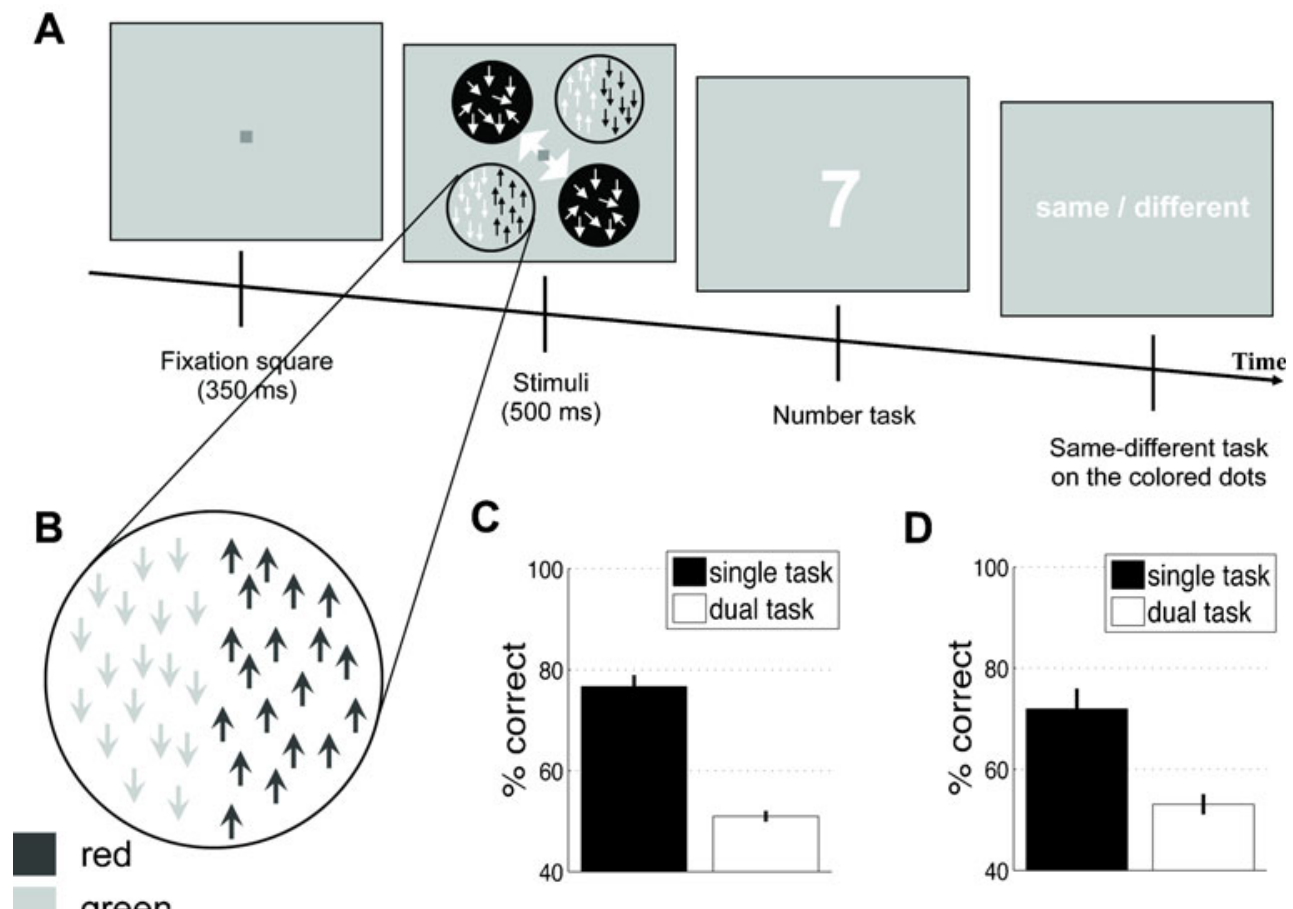

Fig. 5 Experiments 3 and 4: Task and results. (a) We used identical stimuli for Experiments 3 and 4 . The task was similar to that of Experiment 2, except that the colored circles now had moving red and green dots substituted for them. (b) We have zoomed in on the stimulus presented in the uncued locations. The left half of the circle contained green or red dots that moved either up or down. The right side of the stimulus contained dots of the other color that moved in the opposite direction. Subjects needed to indicate whether or not the red dots moved in the same direction in the two uncued circles. (c) Results from Experiment 3. When performed in isolation, the same-different task on the colored dot motion was manageable for the subjects, who performed at about 78\% correct ("single task" bar). However, when subjects were required to concurrently perform the number task from Experiment 1, performance dropped to chance level ("dual task" bar). (d) Results from Experiment 4. This experiment was identical to Experiment 3, except that the two tasks (see panel a) were presented in random order, such that on about half of the trials subjects were asked about the uncued stimuli first. Subjects were again good at the same-different task when they did not need to perform the number task, but dropped to chance level in the main task of the experiment. These results suggest that there was little spill of attentional resources to the uncued location during the main task of Experiment 1 
diagonal equally well in the two experiments $[t(26)=0.53$, $p=.60]$, and they performed equally well on the number task for both $[t(26)=1.08, p=.29]$. The only difference came from the RTs, which were higher in Experiment 3 (mean $\mathrm{RT}=1.21 \mathrm{~s}$ ) than in Experiment 1 (mean $\mathrm{RT}=0.84 \mathrm{~s})[t(26)$ $=2.51, p=.02]$. As in Experiment 2, this difference was to be expected, given that subjects were performing a dual task in Experiment 3 and a single task in Experiment 1.

Critically, subjects were able to indicate whether the patterns of color motion were the same in the two circles or different (i.e., whether or not the red dots moved in the same direction) when they attended only to these stimuli $[t$ $(8)=11.68, p<.0001]$. However, when subjects were required to simultaneously perform the number task, they were no longer able to identify the colored motion [mean accuracy $=50.98 \%$ correct; $t(8)=0.91, p=.38$; see Fig. $5 \mathrm{c}]$.

It should be noted that the weak motion in Experiment 1 (coherence $=4 \%$ ) had much lower coherence than did the colored moving dots (mean coherence $=29 \%, S D=10 \%$ ), and thus should have attracted less exogenous attention. In other words, Experiment 3 provided a conservative control for the amounts of both endogenous and exogenous attention devoted to the uncued locations. Nevertheless, the results still showed that even when subjects were paying more exogenous and endogenous attention to the uncued stimuli (in Exp. 3), this attention was still not enough to complete at a better-than-chance level a task that critically depended on attention. This suggests that the uncued stimuli in Experiment 1 were indeed operating in the near absence of attention.

\section{Experiment 4}

Experiments 2 and 3 suggested that in the paradigm in Experiment 1, subjects were unlikely to have been able to pay focused attention to the uncued stimuli. Both experiments provided conservative tests for this conclusion since, unlike the uncued motion in Experiment 1, the colored stimuli in Experiments 2 and 3 were task relevant.

Nevertheless, one remaining concern is that the influence of the uncued stimuli might have been present at the time when the subjects completed the number task but could have vanished by the time they were answering the same-different question on the colored stimuli. Thus, if the influence of the colored stimuli was short-lived, subjects could have performed the same-different task at a chance level even though they potentially had processed the stimuli enough to perform better than chance if asked about the stimuli before the presentation of the number task. To test for this possibility, in Experiment 4 we used the same design as in Experiment 3 but randomized the order of the number task and the same-different task on the colored stimuli. To anticipate, we found that subjects were still at chance on the same-different task, even though it came before the number question on half of the trials.

Method

Subjects A group of 9 students from the Columbia University undergraduate population ( 3 women, 6 men; mean age $=26.7$ years, range $=20-39)$ participated in these experiment and were paid $\$ 10$ for approximately $1 \mathrm{~h}$ of participation. No subject had participated in any of Experiments 1-3. All subjects had normal or corrected-to-normal vision and signed an informed-consent statement.

Materials and procedure The stimulus presentation was identical to that in Experiment 3. We employed the moving red- and green-dot pattern in the uncued locations (Fig. 5a). To summarize, the experiment began with training of the "color task," in which the coherence of the colored dots was adjusted to achieve performance of at least $70 \%$ accuracy. We proceeded with the calibration procedure that ensured that subjects could correctly identify the direction of motion in the cued diagonal on at least $90 \%$ of trials. The main task was the same as in Experiments 1-3. Subjects needed to do the number task on the basis of the direction of motion in the cued diagonal and to complete the same-different task. Critically, the order of these two tasks was randomized in order to test whether subjects would be able to perform a task on the colored stimuli without a delay between the stimuli and the task.

\section{Results and discussion}

As in Experiments 1-3 we minimized the effects of trials with extreme RTs by excluding trials with RTs below 250 $\mathrm{ms}$ and above $8 \mathrm{~s}$ (resulting in $6.4 \%$ excluded trials) and using median rather than mean RTs in our analyses.

As in Experiments 2 and 3, we tested whether there were significant differences between Experiments 1 and 4 in the main task. Comparing Experiments 1 and 4, we found that the coherence of the cued motion was similar across the two experiments $[t(26)=0.17, p=.86]$ and that subjects were able to judge the direction of motion in the cued diagonal equally well in the two experiments $[t(26)=0.34, p=.74]$. However, subjects performed worse on the number task in Experiment 4 (average $d^{\prime}=1.71$ ) than in Experiment 1 (average $\left.d^{\prime}=2.8\right)[t(26)=2.48, p=.02]$, and their RTs were higher in Experiment 4 (mean RT $=1.08 \mathrm{~s}$ ) than in Experiment 1 (mean RT $=0.84 \mathrm{~s}$ ), with this difference approaching significance $[t(26)=1.91, p=.07]$. As in Experiments 2 and 3, this difference was to be expected, given that subjects were performing a dual task in Experiment 4 and a single task in Experiment 1. 
As in Experiment 3, subjects were able to indicate whether the patterns of color motion were the same in the two circles (i.e., whether or not the red dots moved in the same direction) when they attended only to these stimuli $[t(8)=$ $5.43, p<.0001]$. However, when subjects were required to simultaneously perform the number task, they were no longer able to identify the colored motion [mean accuracy $=$ $53.08 \%$ correct; $t(8)=1.54, p=.16$; see Fig. $5 \mathrm{~d}]$, despite the fact that on half of the trials the question about the colored stimuli came immediately after presentation of the stimuli.

As in Experiment 3, the weak motion in Experiment 1 (coherence $=4 \%$ ) had much lower coherence than did that of the colored moving dots (mean coherence $=32 \%, S D=$ $7 \%$ ), and thus should have attracted less exogenous attention. In other words, Experiment 4 provided a conservative control for the amounts of both endogenous and exogenous attention devoted to the uncued locations.

\section{General discussion}

We investigated the influence of subliminal stimuli on higher cognitive processes in the near absence of spatial attention. We demonstrated that stimuli (i.e., moving dots) outside of the attentional focus could influence task preparation and execution, one of the highest forms of cognitive control known to depend on the prefrontal cortex. The effect was present even when we interpolated the point at which subjects were only able to identify the uncued stimuli at chance level. To the best of our knowledge, this is the first demonstration that subliminal stimuli outside of the focus of endogenous spatial attention can influence cognitive control.

Our results are at odds with several influential theories about attention and consciousness (Dehaene et al., 2006; Jack \& Shallice, 2001; Koch \& Tsuchiya, 2007; Norman $\&$ Shallice, 1986). In particular, Koch and Tsuchiya created a taxonomy of behaviors that are possible without attention and/or consciousness. In their taxonomy, priming is placed among processes that do not require consciousness but do require attention. Our results challenge this placement and suggest that priming should be among the processes that do not require either consciousness or focused attention. In a competing taxonomy, Dehaene et al. (2006) suggested that events can be divided into conscious, preconscious, and subliminal. According to that taxonomy, our stimuli would be subliminal, since their bottom-up stimulus strength is weak and they do not receive focused attention. Dehaene et al. predicted that unattended subliminal stimuli should produce little or no priming and should not reliably excite the frontoparietal network necessary for priming of cognitive control. Our results challenge this prediction, too.
One important issue will be to determine the depth of processing of the uncued motion. Previous research has demonstrated that the presence of cuing effects does not necessarily demonstrate manipulation of the highest control center in the brain (Logan \& Bundesen, 2003). With this caveat in mind, what we claim here is that the effect we observed probably operated via cognitive processes beyond the early perceptual level. Future studies using brainimaging methods (Lau \& Passingham, 2007; van Gaal et al., 2008; van Gaal et al., 2010) will be needed to conclusively resolve this issue.

How does subliminal uncued motion influence task set? Traditionally, it has been assumed that both conscious perception and executive control functions (e.g., task-set preparation) depend on activity in the prefrontal cortex (Koechlin et al., 2003; Miller \& Cohen, 2001). However, recent imaging studies (Lau \& Passingham, 2007; van Gaal et al., 2008; van Gaal et al., 2010) have found that executive functions triggered by unconscious stimuli also activated the prefrontal cortex. The fact that unconscious stimuli can access neuronal resources in this region seems to go against theories that postulate that the prefrontal cortex is critical for consciousness, such as the global workspace theory (Dehaene \& Naccache, 2001). However, we have recently argued that, though consciousness may indeed depend on prefrontal mechanisms, such mechanisms may be specifically related to perceptual decision making and sensory metacognition and may be distinct from other prefrontal mechanisms, such as those that support executive control functions (Lau \& Rosenthal, 2011). Thus, subliminal uncued motion could be able to access executive control functions without leading to conscious perception, even though both may depend on anatomically proximal (but distinct) regions in the prefrontal cortex.

Interestingly, another line of research has suggested that unconscious priming is impossible without temporal attention (Fabre, Lemaire, \& Grainger, 2007; Kiefer \& Brendel, 2006; Naccache, Blandin, \& Dehaene, 2002). This finding is in apparent contradiction with our findings that unconscious priming is possible without endogenous spatial attention. However, temporal and spatial attention have previously been shown to have differential effects on some forms of priming. In particular, Fabre et al. reported that temporal attention is needed for categorical but not for repetition priming. On the other hand, other studies have reported that spatial attention is needed for repetition priming (Besner et al., 2005; Lachter et al., 2004; Marzouki et al., 2007). Our paradigm could potentially help further elucidate the differences between spatial and temporal attention.

One alternative explanation for the difference between our study and the previous ones that did not find priming without attention is that the crucial difference lies in the 
types of stimuli used. A recent study (Finkbeiner \& Palermo, 2009) showed that even though such stimuli as words, letters, or numbers are only able to prime in the presence of attention (Fabre et al., 2007; Kiefer \& Brendel, 2006; Naccache et al., 2002), faces can prime even without attention. Finkbeiner and Palermo argued that the difference might lie in the fact that the former stimuli are processed by evolutionarily newer brain regions that might be more dependent on attention than are the evolutionarily old regions that process faces. Our motion stimuli are also processed mainly in evolutionarily old regions of the brain (Striedter, 2005). Another possibility is that motion processing depends more on the dorsal than on the ventral visual pathway, while such static stimuli as words show the reverse pattern of dependence (Mishkin \& Ungerleider, 1982). It is likely that attentional processing might be different for the dorsal and ventral pathways. Regardless of the exact reason for the difference between the present and past studies, it should be noted that unlike the previous research, we report priming of cognitive control, which implies that our stimuli were necessarily processed beyond the primary sensory areas.

Finally, one critical issue is whether the uncued stimuli in our experiment received no spatial attention whatsoever. We do not argue for such a strong position, and do not think that this issue could be resolved easily at present. The uncued stimuli were weak and irrelevant to the task, so subjects had no reason to attend to them. In our control experiments, we presented stimuli that, unlike natural scenes or faces, required spatial attention to be identified (Lee et al., 1999; Li et al., 2002; Reddy, Moradi, \& Koch, 2007). At the uncued locations, subjects could not identify such stimuli when they had to perform the main task, which suggests that the subjects could not have deployed a substantial amount of attentional resources to the uncued locations. Using a similar procedure, previous researchers have argued that such stimuli operated in the "near absence of attention" ( $\mathrm{Li}$ et al., 2002). Following this characterization, then, our results represent a first step in demonstrating the possibility that subliminal stimuli may influence cognitive control, even when they are presented at locations that receive a "near absence" of endogenous spatial attention. A complete theory of attention and cognitive control should account for how this could be possible.

Author Note We thank Floris de Lange and Dick Passingham for helpful comments. H.L. received funding from the Human Frontiers Science Project (HFSP Short-Term Fellowship) and the Templeton Foundation (Grant 15462-SCI04). The authors declare no conflict of interest.

Open Access This article is distributed under the terms of the Creative Commons Attribution Noncommercial License which permits any noncommercial use, distribution, and reproduction in any medium, provided the original author(s) and source are credited.

\section{References}

Baars, B. J. (2002). The conscious access hypothesis: Origins and recent evidence. Trends in Cognitive Sciences, 6, 47-52.

Bahrami, B., Lavie, N., \& Rees, G. (2007). Attentional load modulates responses of human primary visual cortex to invisible stimuli. Current Biology, 17, 509-513. doi:10.1016/j.cub.2007.01.070

Barnett, V., \& Lewis, T. (1994). Outliers in statistical data (3rd ed.). New York, NY: Wiley.

Besner, D., Risko, E. F., \& Sklair, N. (2005). Spatial attention as a necessary preliminary to early processes in reading. Canadian Journal of Experimental Psychology, 59, 99-108.

Brainard, D. H. (1997). The Psychophysics Toolbox. Spatial Vision, 10, 433-436. doi:10.1163/156856897X00357

Dehaene, S., Changeux, J.-P., Naccache, L., Sackur, J., \& Sergent, C. (2006). Conscious, preconscious, and subliminal processing: A testable taxonomy. Trends in Cognitive Sciences, 10, 204-211. doi:10.1016/j.tics.2006.03.007

Dehaene, S., \& Naccache, L. (2001). Towards a cognitive neuroscience of consciousness: Basic evidence and a workspace framework. Cognition, 79, 1-37. doi:10.1016/S0010-0277(00)00123-2

Dehaene, S., Naccache, L., Le Clec'H, G., Koechlin, E., Mueller, M., Dehaene-Lambertz, G., . . . Le Bihan, D. (1998). Imaging unconscious semantic priming. Nature, 395, 597-600. doi:10.1038/ 26967

Dijksterhuis, A., Bos, M. W., Nordgren, L. F., \& van Baaren, R. B. (2006). On making the right choice: The deliberation-withoutattention effect. Science, 311, 1005-1007.

Eimer, M., \& Schlaghecken, F. (2003). Response facilitation and inhibition in subliminal priming. Biological Psychology, 64, 7-26.

Fabre, L., Lemaire, P., \& Grainger, J. (2007). Attentional modulation of masked repetition and categorical priming in young and older adults. Cognition, 105, 513-532.

Ferguson, M. J., \& Bargh, J. A. (2004). How social perception can automatically influence behavior. Trends in Cognitive Sciences, 8 , 33-39.

Finkbeiner, M., \& Palermo, R. (2009). The role of spatial attention in nonconscious processing: A comparison of face and nonface stimuli. Psychological Science, 20, 42-51.

Finlay, D. (1982). Motion perception in the peripheral visual field. Perception, 11, 457-462.

Greenwald, A. G., Draine, S. C., \& Abrams, R. L. (1996). Three cognitive markers of unconscious semantic activation. Science, 273, 1699-1702. doi:10.1126/science.273.5282.1699

Jack, A. I., \& Shallice, T. (2001). Introspective physicalism as an approach to the science of consciousness. Cognition, 79, 161196.

Jonides, J. (1981). Voluntary versus automatic control over the mind's eye's movement. In J. Long \& A. Baddeley (Eds.), Attention and performance $I X$ (pp. 187-203). Hillsdale, NJ: Erlbaum.

Kiefer, M., \& Brendel, D. (2006). Attentional modulation of unconscious "automatic" processes: Evidence from event-related potentials in a masked priming paradigm. Journal of Cognitive Neuroscience, 18, 184-198.

Koch, C. (2004). The quest for consciousness: A neurobiological approach. Englewood, CO: Roberts \& Co.

Koch, C., \& Tsuchiya, N. (2007). Attention and consciousness: Two distinct brain processes. Trends in Cognitive Sciences, 11, 16-22.

Koechlin, E., Ody, C., \& Kouneiher, F. (2003). The architecture of cognitive control in the human prefrontal cortex. Science, 302, 1181-1185. doi:10.1126/science.1088545

Kouider, S., \& Dehaene, S. (2007). Levels of processing during non-conscious perception: A critical review of visual masking. Philosophical Transactions of the Royal Society B, 362, 857875. 
Lachter, J., Forster, K. I., \& Ruthruff, E. (2004). Forty-five years after Broadbent (1958): Still no identification without attention. Psychological Review, 111, 880-913. doi:10.1037/0033-295X.111.4.880

Lau, H. C., \& Passingham, R. E. (2007). Unconscious activation of the cognitive control system in the human prefrontal cortex. Journal of Neuroscience, 27, 5805-5811.

Lau, H., \& Rosenthal, D. (2011). Empirical support for higher-order theories of conscious awareness. Trends in Cognitive Sciences, $15,365-373$.

Lee, D. K., Koch, C., \& Braun, J. (1999). Attentional capacity is undifferentiated: Concurrent discrimination of form, color, and motion. Perception \& Psychophysics, 61, 1241-1255.

Li, F. F., VanRullen, R., Koch, C., \& Perona, P. (2002). Rapid natural scene categorization in the near absence of attention. Proceedings of the National Academy of Sciences, 99, 9596-9601.

Logan, G. D., \& Bundesen, C. (2003). Clever homunculus: Is there an endogenous act of control in the explicit task-cuing procedure? Journal of Experimental Psychology: Human Perception and Performance, 29, 575-599. doi:10.1037/00961523.29.3.575

Marzouki, Y., Grainger, J., \& Theeuwes, J. (2007). Exogenous spatial cueing modulates subliminal masked priming. Acta Psychologica, $126,34-45$.

Mattler, U. (2003). Priming of mental operations by masked stimuli. Perception \& Psychophysics, 65, 167-187.

McMullen, P. A., MacSween, L. E., \& Collin, C. A. (2009). Behavioral effects of visual field location on processing motion- and luminance-defined form. Journal of Vision, 9, 24(6), 1-11. doi: $10.1167 / 9.6 .24$

Miller, E. K., \& Cohen, J. D. (2001). An integrative theory of prefrontal cortex function. Annual Review of Neuroscience, 24, 167-202. doi:10.1146/annurev.neuro.24.1.167

Mishkin, M., \& Ungerleider, L. G. (1982). Contribution of striate inputs to the visuospatial functions of parieto-preoccipital cortex in monkeys. Behavioural Brain Research, 6, 57-77.

Moreland, J. D., Jameson, D., \& Hurvich, L. M. (1972). Peripheral colour vision. In Handbook of sensory physiology: Vol.
VII/4. Visual psychophysics (pp. 517-536). New York, NY: Springer.

Naccache, L., Blandin, E., \& Dehaene, S. (2002). Unconscious masked priming depends on temporal attention. Psychological Science, 13, 416-424. doi:10.1111/1467-9280.00474

Norman, D. A., \& Shallice, T. (1986). Attention to action: Willed and automatic control of behaviour. Consciousness and SelfRegulation, 4, 1-18.

Reddy, L., Moradi, F., \& Koch, C. (2007). Top-down biases win against focal attention in the fusiform face area. NeuroImage, 38, 730-739.

Sakai, K., \& Passingham, R. E. (2003). Prefrontal interactions reflect future task operations. Nature Neuroscience, 6, 75-81.

Striedter, G. F. (2005). Principles of brain evolution. Sunderland, MA: Sinauer.

Umiltà, C. (1988). The control operations of consciousness. In A. J. Marcel \& E. Bisiach (Eds.), Consciousness in contemporary science (pp. 334-356). Oxford: Oxford University Press.

van Gaal, S., Ridderinkhof, K. R., Fahrenfort, J. J., Scholte, H. S., \& Lamme, V. A. F. (2008). Frontal cortex mediates unconsciously triggered inhibitory control. Journal of Neuroscience, 28, 80538062. doi:10.1523/JNEUROSCI.1278-08.2008

van Gaal, S., Ridderinkhof, K. R., Scholte, H. S., \& Lamme, V. A. F. (2010). Unconscious activation of the prefrontal no-go network. Journal of Neuroscience, 30, 4143-4150. doi:10.1523/JNEUROSCI.2992-09.2010

van Gaal, S., Ridderinkhof, K. R., van den Wildenberg, W. P. M., \& Lamme, V. A. F. (2009). Dissociating consciousness from inhibitory control: Evidence for unconsciously triggered response inhibition in the stop-signal task. Journal of Experimental Psychology: Human Perception and Performance, 35, 11291139. doi: $10.1037 / \mathrm{a} 0013551$

Varraine, E., Bonnard, M., \& Pailhous, J. (2002). Interaction between different sensory cues in the control of human gait. Experimental Brain Research, 142, 374-384.

Wolfe, J. M., \& Horowitz, T. S. (2004). What attributes guide the deployment of visual attention and how do they do it? Nature Reviews Neuroscience, 5, 495-501. doi:10.1038/nrn1411 